\title{
Conductivity and permittivity of dispersed systems with penetrable particle-host interphase
}

\author{
M.Ya. Sushko, A.K. Semenov \\ Mechnikov National University, Department of Theoretical Physics, 2 Dvoryanska St., 65026 Odesa, Ukraine
}

Received July 3, 2012, in final form November 28, 2012

A model for the study of the effective quasistatic conductivity and permittivity of dispersed systems with particlehost interphase, within which many-particle polarization and correlation contributions are effectively incorporated, is presented. The structure of the system's components, including the interphase, is taken into account through modelling their low-frequency complex permittivity profiles. The model describes, among other things, a percolation-type behavior of the effective conductivity, accompanied by a considerable increase in the real part of the effective complex permittivity. The percolation threshold location is determined mainly by the thickness of the interphase. The "double" percolation effect is predicted. The results are contrasted with experiment.

Key words: core-shell particle, dispersion, permittivity, conductivity, percolation

PACS: 42.25.Dd, 64.60.Ak, 77.22.Ch, 82.70.-y, 83.80. Hj

\section{Introduction}

The studies of disperse systems, nanofluids, and systems of nanoparticles are nontrivial, but important for many fields in science and industry. Much attention has been recently focused on the question how the properties of the particle-host interphase affect the dielectric characteristics of the entire system [1-4]. This is an essentially many-particle problem, playing a crucial role, say, in percolation studies [5-7]. The latter involve (a) various numerical (mainly Monte-Carlo) methods [8, 9], (b) graph [10] and renormalization group [7] theories, (c) different improvements of the Maxwell-Garnett [11] and Bruggeman [12] approaches.

It should be noted that the practical realization of methods (a) is labor-consuming and imposes high computer specifications. Methods (b) provide certain scaling relations, but ignore the physical nature of dielectric response of an inhomogeneous system. Methods (c) seem more adequate physically, but are actually one-particle approaches. They can demonstrate a percolation-type behavior of conductivity [13], but ignore the fractal nature of percolation cluster [14]. Numerous attempts at improving these approaches are widespread in modern technical literature (see [15]), but are, for most part, poorly justified physically.

In the present work, the role of the interphase in the formation of a percolation-type behavior of conductivity is analyzed within an electrodynamic model based upon the method of compact groups of inhomogeneities [16-18]. The “compact group” means any macroscopic region, in which all distances between the inhomogeneities are much shorter than the wavelength of probing radiation in the medium. The method allows one to effectively sum up and average the electric field and displacement vectors in finely-dispersed systems of particles with complex internal structure and arbitrary permittivities. In doing so, unnecessary detailed elaboration of the processes of interparticle polarization and correlations in the system is avoided.

The main idea of the method is that in the long-wave limit, a macroscopically homogeneous and isotropic particulate system is electrodynamically equivalent to an aggregate of compact groups of particles. The compact groups include macroscopic numbers of particles and can be viewed as one-point 
inhomogeneities, with negligibly small particle number fluctuations inside. They are in fact self-similar. Note that this property is characteristic of a percolation cluster.

The model of the disperse system under consid-

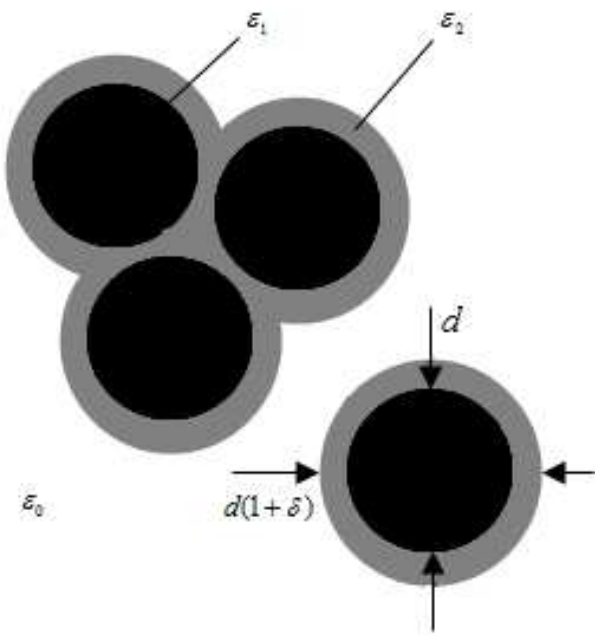

Figure 1. The model under consideration. eration is depicted in figure 1. Each particle consists of a spherical hard core (black), with diameter $d$ and permittivity $\varepsilon_{1}$. The core is surrounded by a penetrable concentric shell (gray), with outer diameter $d(1+\delta)$ ( $\delta$ being the relative thickness of the shell) and permittivity $\varepsilon_{2}$. The particles are embedded into a host medium (white), with permittivity $\varepsilon_{0}$. All the permittivities are in general complex. The volume concentration of the hard-cores is $c$. The shells can freely overlap; together, they form the interphase between the hard cores and the host. The volume concentration $\phi_{\text {int }}$ of the interphase for particular values $c$ and $\delta$ can be calculated by statistical methods. We take it from Monte Carlo simulations [19, 20].

We expect that, in the main features, our results should remain valid for macroscopically homogeneous and isotropic systems with non-spherical core-shell particles: the sphericity comes into play at the points where the explicit form of the function $\phi_{\text {int }}=\phi_{\text {int }}(c, \delta)$ is needed. By contrast, spherical symmetry of the particles is crucial for most approaches to the problem which often represent different versions of one- and two-particle approximations and use it either implicitly or through the requirement that the shells should be hard. On the other hand, the systems of anisotropic particles are usually treated as if the latter were solitary. Some examples and further literature on these topics can be found in $[4,18,21,22]$.

\section{General equation for the low-frequency complex permittivity}

The structure of the system's components, including the interphase, is taken into account through modelling their complex permittivity profiles and the choice of the way of homogenization.

We are interested in the low-frequency value $\varepsilon=\varepsilon^{\prime}-\mathrm{i} 4 \pi \sigma / \omega$ of the effective complex permittivity and determine it as the proportionality coefficient in the relation

$$
\langle\mathbf{D}(\mathbf{r})\rangle=\langle\epsilon(\mathbf{r}) \mathbf{E}(\mathbf{r})\rangle=\varepsilon\langle\mathbf{E}(\mathbf{r})\rangle,
$$

where $\mathbf{D}(\mathbf{r}), \mathbf{E}(\mathbf{r})$, and $\epsilon(\mathbf{r})$ are the local values of, respectively, the electric displacement, electric field, and permittivity in the system, and the angular brackets stand for the statistical averaging. In the present work, we suggest that this averaging can be reduced to the following two-step procedure.

1. Finding the equilibrium distribution of the particles with penetrable outer shells. This distribution is characterized by the hard-cores' volume concentration $c$ and the particles' effective volume concentration $\phi=\phi(c, \delta)$ (the latter is the sum of $c$ and the volume concentration of the regions occupied by the shells). Electrodynamically, such a system can be viewed as an aggregate of non-overlapping (white, black, and gray in figure 1 regions with permittivities $\varepsilon_{i}=\varepsilon_{i}^{\prime}-\mathrm{i} 4 \pi \sigma_{i} / \omega$, $i=0,1,2$, and volume concentrations $1-\phi, c$, and $\phi-c$, respectively.

2. Electrodynamic homogenization, by direct integration with respect to its volume [23], of this aggregate within the compact group approach. This suggests that: (a) with respect to a probing field of long wavelength $\lambda$, the aggregate itself is viewed as a set of regions (termed as compact groups) 
with typical linear sizes much smaller than $\lambda$, but yet remaining macroscopic; (b) the macroscopic dielectric properties of the aggregate are equivalent to those of a macroscopically homogeneous and isotropic system prepared by embedding the aggregate's non-overlapping regions into some fictitious medium, of permittivity $\epsilon$. The permittivity distribution in this fictitious system can be written as

$$
\epsilon(\mathbf{r})=\epsilon+\delta \epsilon(\mathbf{r})
$$

where, in our case,

$$
\delta \epsilon(\mathbf{r})=\left(\varepsilon_{0}-\epsilon\right) \Pi_{0}\left(\mathbf{r}, \Omega_{0}\right)+\sum_{a=1}^{N}\left(\varepsilon_{1}-\epsilon\right) \Pi_{1}\left(\mathbf{r}, \Omega_{a}\right)+\sum_{b=1}^{N^{\prime}}\left(\varepsilon_{2}-\epsilon\right) \Pi_{2}\left(\mathbf{r}, \Omega_{b}^{\prime}\right)
$$

is the local permittivity deviation from $\epsilon$ due to the presence of a compact group of the aggregate's non-overlapping regions at point $\mathbf{r}$. Here, $\Pi_{0}\left(\mathbf{r}, \Omega_{0}\right), \Pi_{1}\left(\mathbf{r}, \Omega_{a}\right)$, and $\Pi_{2}\left(\mathbf{r}, \Omega_{b}^{\prime}\right)$ are the characteristic functions of the regions $\Omega_{0}, \Omega_{a}$, and $\Omega_{b}^{\prime}$ occupied by, respectively, the real host, the $a$ th particle's hard core, and the $b$ th connected cluster of the overlapping outer shells:

$$
\Pi_{i}(\mathbf{r}, \Omega)= \begin{cases}1, & \mathbf{r} \in \Omega, \\ 0, & \mathbf{r} \notin \Omega .\end{cases}
$$

With the model permittivity distribution (2.2) and (2.3) proposed, the averaged displacement and field in formula (2.1) are calculated by the general formulae

$$
\begin{gathered}
\langle\mathbf{E}\rangle=\left\{1+\sum_{s=1}^{\infty}\left(-\frac{1}{3 \epsilon}\right)^{s}\left\langle[\delta \epsilon(\mathbf{r})]^{s}\right\rangle\right\} \mathbf{E}_{0}, \\
\langle\mathbf{D}\rangle=\left\{\epsilon+\epsilon \sum_{s=1}^{\infty}\left(-\frac{1}{3 \epsilon}\right)^{s}\left\langle[\delta \epsilon(\mathbf{r})]^{s}\right\rangle+\sum_{s=0}^{\infty}\left(-\frac{1}{3 \epsilon}\right)^{s}\left\langle[\delta \epsilon(\mathbf{r})]^{s+1}\right\rangle\right\} \mathbf{E}_{0} .
\end{gathered}
$$

In view of the property 2.4, the averages

$$
\left\langle[\delta \varepsilon(\mathbf{r})]^{n}\right\rangle=\frac{1}{V} \int_{V}[\delta \varepsilon(\mathbf{r})]^{n} \mathrm{~d} \mathbf{r}
$$

are easy to find:

$$
\left\langle[\delta \epsilon(\mathbf{r})]^{n}\right\rangle=(1-\phi)\left(\varepsilon_{0}-\epsilon\right)^{n}+c\left(\varepsilon_{1}-\epsilon\right)^{n}+(\phi-c)\left(\varepsilon_{2}-\epsilon\right)^{n} .
$$

Substitution of (2.7) into series [2.5), (2.6) and summation of those give

$$
(1-\phi) \frac{\varepsilon_{0}-\epsilon}{2 \epsilon+\varepsilon_{0}}+c \frac{\varepsilon_{1}-\epsilon}{2 \epsilon+\varepsilon_{1}}+(\phi-c) \frac{\varepsilon_{2}-\epsilon}{2 \epsilon+\varepsilon_{2}}=\frac{\varepsilon-\epsilon}{2 \epsilon+\varepsilon} .
$$

The permittivity $\epsilon$ is the only unknown parameter left. In what follows, we suggest that the dielectric properties of the fictitious and effective systems are identical, that is, $\epsilon=\varepsilon$. This approximation is equivalent to the Bruggeman-type homogenization, when the host and dispersed particles are treated symmetrically. This seems reasonable for the system under study because the shape of the white and gray regions is extremely complicated. The degree of this complexity increases with $c$ even more.

The other extreme case, $\epsilon=\varepsilon_{0}$, corresponds to the Maxwell-Garnett type of homogenization.

\section{Main features of the model}

In the quasistatic limit and at $\epsilon=\varepsilon$, the general equation (2.8) reduces to two real equations, a cubic one for $\sigma$ and, once $\sigma$ is found, a linear for $\varepsilon^{\prime}$ :

$$
(1-\phi) \frac{\sigma_{0}-\sigma}{2 \sigma+\sigma_{0}}+c \frac{\sigma_{1}-\sigma}{2 \sigma+\sigma_{1}}+(\phi-c) \frac{\sigma_{2}-\sigma}{2 \sigma+\sigma_{2}}=0 .
$$




$$
(1-\phi) \frac{\varepsilon_{0}^{\prime} \sigma-\varepsilon^{\prime} \sigma_{0}}{\left(2 \sigma+\sigma_{0}\right)^{2}}+c \frac{\varepsilon_{1}^{\prime} \sigma-\varepsilon^{\prime} \sigma_{1}}{\left(2 \sigma+\sigma_{1}\right)^{2}}+(\phi-c) \frac{\varepsilon_{2}^{\prime} \sigma-\varepsilon^{\prime} \sigma_{2}}{\left(2 \sigma+\sigma_{2}\right)^{2}}=0 .
$$

A general analytical analysis of equation (3.1) can be carried out with the use of Cardano's formulas and is cumbersome [24]. Nonetheless, the major features of the model can be grasped with simple physical reasoning. For the sake of convenience, we change in equations (3.1) and (3.2) to dimensionless variables $x \equiv \sigma / \sigma_{1}, y=\varepsilon^{\prime} / \varepsilon_{0}^{\prime}$ and $x_{i} \equiv \sigma_{i} / \sigma_{1}, y_{i}=\varepsilon_{i}^{\prime} / \varepsilon_{0}^{\prime}(i=0,1,2)$. Typically, $x_{0} \ll 1$ and $y>1$.

\subsection{Percolation-type behavior}

In the limit of a non-conducting matrix, $x_{0} \rightarrow 0$, equation (3.1) has three solutions, $x=0$ and

$$
x=\frac{3}{4}\left\{\left(c-\frac{1}{3}\right)+\left(\phi-\frac{1}{3}-c\right) x_{2} \pm \sqrt{\frac{4}{3}\left(\phi-\frac{1}{3}\right) x_{2}+\left[\left(c-\frac{1}{3}\right)+\left(\phi-\frac{1}{3}-c\right) x_{2}\right]^{2}}\right\}
$$

If $x_{2}>0$, a physically meaningful nontrivial solution (that with the positive sign in front of the square root, see figure 2) appears only under the condition

$$
\phi\left(c_{\mathrm{c}}, \delta\right)=\frac{1}{3}
$$

and is independent of $x_{2}$. In the case $x_{2}=0$, the well-known value of $c_{\mathrm{c}}=1 / 3$ for the two-component Bruggeman model occurs [13].

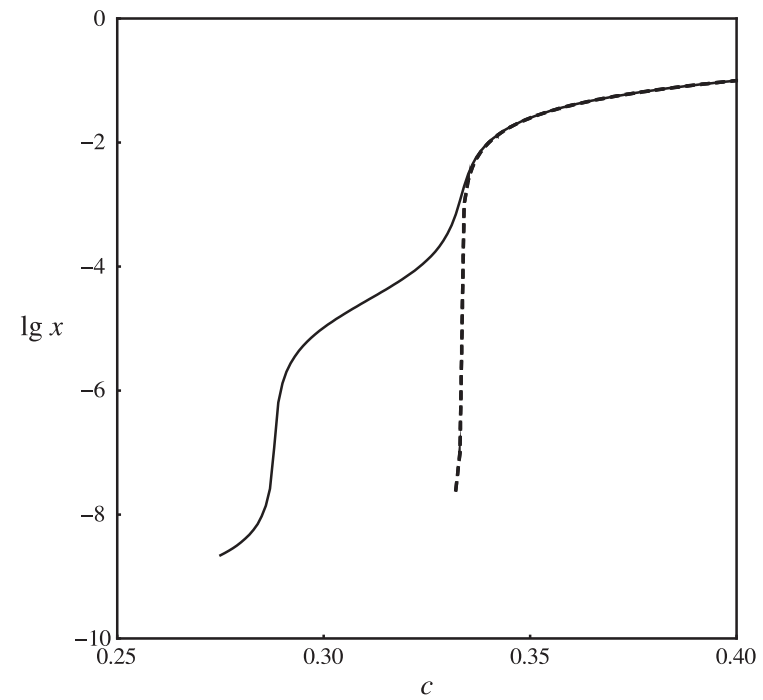

Figure 2. Percolation (dashed line, $\delta=0$ ) and "double" percolation (solid line, $\delta=0.05$ ); $x_{0}=1 \cdot 10^{-10}$, $x_{2}=5 \cdot 10^{-5}$.

The relation (3.4) determines the threshold concentration $c_{\mathrm{c}}$ for the effective conductivity. This value is affected only by the fact of the existence of the interphase $(\delta \neq 0)$, rather than its dielectric and conductive properties.

Our estimate of $c_{\mathrm{c}}$ as a function of the relative thickness $\delta$ of the interphase layer is shown in figure 3 The calculations were performed using the Monte Carlo results [20] $\left(k=(1+\delta)^{-3}, \varphi=c / k\right)$ :

$$
\begin{aligned}
\phi= & 1-(1-k \varphi) \exp [-(1-k) \varphi] \exp \left\{-\frac{k \varphi^{2}}{2(1-k \varphi)^{3}}\left[\left(8-9 k^{1 / 3}+k\right)\right.\right. \\
& \left.\left.-\left(4+9 k^{1 / 3}-18 k^{2 / 3}+5 k\right) k \varphi+2(1-k) k^{2} \varphi^{2}\right]\right\} .
\end{aligned}
$$




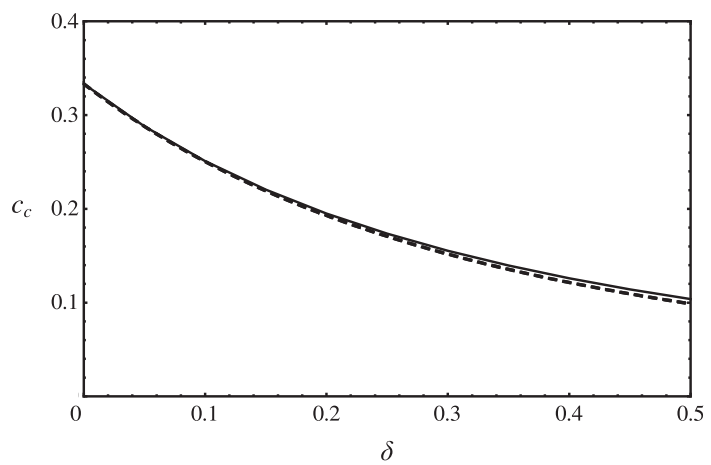

Figure 3. Threshold concentration as a function of the relative thickness of the interphase (solid line). Dashed line: $c_{\mathrm{C}} \simeq \frac{1}{3}(1+\delta)^{-3}$.

The analysis revealed that for realistic $c \lesssim 0.5$, the relation $c_{\mathrm{c}} \simeq \frac{1}{3}(1+\delta)^{-3}$ can be used.

In the immediate vicinity of $c_{\mathrm{c}}\left(c \rightarrow c_{\mathrm{c}}+0\right)$ and for nonzero $\delta$, formula 3.3 takes the form

$$
x \simeq \frac{3}{4} x_{2}\left[1+\frac{\frac{1}{3}+c\left(1-x_{2}\right)}{\frac{1}{3}-c\left(1-x_{2}\right)}\right]\left(\phi-\frac{1}{3}\right) .
$$

Correspondingly, the effective conductivity $\sigma \propto\left(c-c_{\mathrm{c}}\right)^{t}$ where the critical exponent $t \simeq 1.0$. The effective permittivity $\varepsilon^{\prime}$, as follows from equation (3.2), increases anomalously as $x_{0} \rightarrow 0$. The latter fact is in accord with predictions [25].

\subsection{Effective critical exponent of conductivity}

In practice, both the threshold concentration $c_{\mathrm{c}}$ and the critical exponent $t$ are determined by interpolating the conductivity experimental data $\sigma=\sigma(c)$, obtained for some finite interval $c \in\left[c_{1}, c_{2}\right]$ near $c_{\mathrm{C}}$ $\left(c_{1}>c_{\mathrm{c}}\right.$ ), with the scaling law $\sigma=A\left(c-c_{\mathrm{c}}\right)^{t}, A$ and $t$ being independent of $c$. Then,

$$
t=\log \frac{\sigma\left(c_{2}\right)}{\sigma\left(c_{1}\right)} / \log \frac{c_{2}-c_{\mathrm{c}}}{c_{1}-c_{\mathrm{c}}},
$$

and this value is expected to be a $c$-independent constant.

By contrast, the asymptotic formula (3.6) reveals that even small variations of $c$ near $c_{\mathrm{c}}$ cause the expression in the brackets, which is proportional to $A$, to change considerably. This means that a formal application of the above procedure and formula (3.7) to a system with conductivity (3.3) will result in an effective exponent $t_{\text {eff }}$ sensitive to the parameters $c_{1}$ and $c_{2}$ (figure 4 ). In particular, for a given $\delta \neq 0, t_{\text {eff }}$

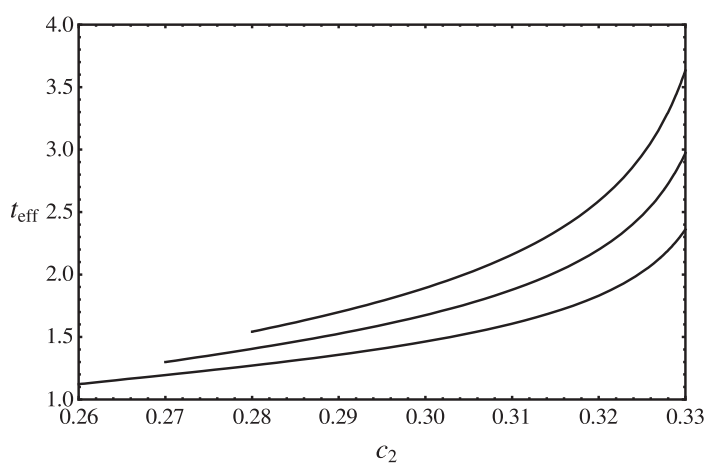

Figure 4. Effective critical exponent of conductivity as a function of $c_{2}$ at fixed $c_{1}, \delta=0.1\left(c_{\mathrm{c}} \simeq 0.251\right.$ ), and $x_{2}=5 \cdot 10^{-5}$, calculated with formulas 3.3 and 3.7. From bottom to top, $c_{1}=0.26,0.27$, and 0.28 . 


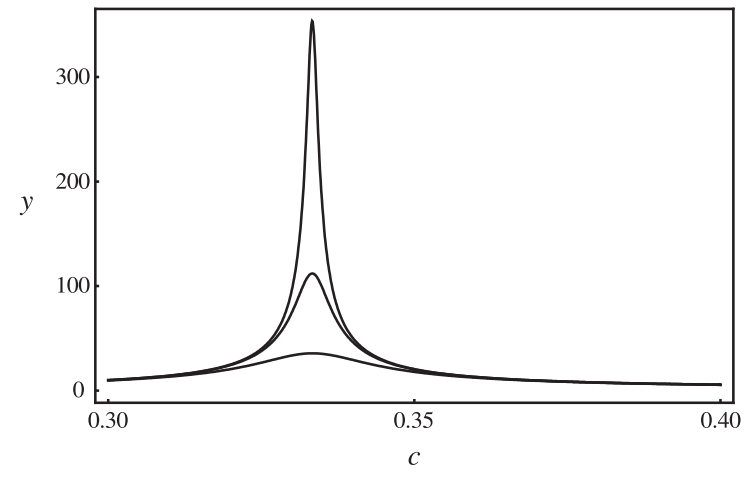

Figure 5. Effect of the matrix's conductivity on the effective permittivity. From top to bottom, $x_{0}=1$. $10^{-6}, 1 \cdot 10^{-5}$, and $1 \cdot 10^{-4}$. The other parameters: $y_{1}=1.5, y_{2}=1, x_{2}=0.05, \delta=0.05$.

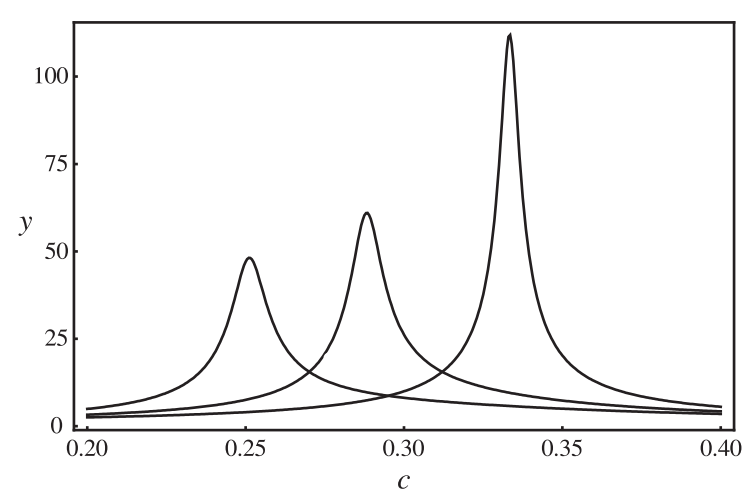

Figure 6. Effect of the interphase thickness on the effective permittivity. From right to left, $\delta=0,0.05$, and 0.10 . The other parameters: $y_{1}=1.5, y_{2}=1$, $x_{0}=1 \cdot 10^{-5}, x_{2}=0.05$.

increases as the interval $\left[c_{1}, c_{2}\right]$ (where $c_{2}<1 / 3$ ) is: (a) shifted to higher values of $c$ (while its width is fixed); (b) widened at fixed $c_{1}$. Also, the threshold concentration found within this procedure is expected to exceed $c_{\mathrm{c}}$.

Different 3D percolation models [5] and renormalization group calculations [26, 27] give for $t$ estimates of $\approx 1.3 \div 1.7$ and $\approx 1.9,2.14$, respectively. Experimental values of $t$ are usually $1.5 \div 2.0$ and sometimes even twice as much [22]. As evident from figure 4 our theory is capable of reproducing a variety of these values.

\subsection{Effect of the matrix's conductivity}

For real substances, $x_{0} \neq 0$, though $x_{0}$ can be extremely small. As a result, the percolation-type dependence of $x$ with $c$ changes to a smooth one, with its slope considerably increasing near $c_{\mathrm{c}}$. Simultaneously, the maximum value of $y$ becomes bounded above and decreases as $x_{0}$ increases (figure 5). The location of the maximum shifts to lower concentrations as $\delta$ increases (figure6). Calculations show that it remains practically independent of $x_{2}$ and actually equal to $c_{\mathrm{c}}$.

Below the percolation threshold, the effective conductivity is usually approximated by the scaling law $\sigma=B\left(c_{\mathrm{c}}-c\right)^{-s}$. Given experimental data for some interval $c \in\left[c_{1}, c_{2}\right], c_{2}<c_{\mathrm{c}}$, the effective values $s_{\mathrm{eff}}$ of the critical exponent $s$ are found as

$$
s=-\log \frac{\sigma\left(c_{2}\right)}{\sigma\left(c_{1}\right)} / \log \frac{c_{\mathrm{c}}-c_{2}}{c_{\mathrm{c}}-c_{1}}
$$

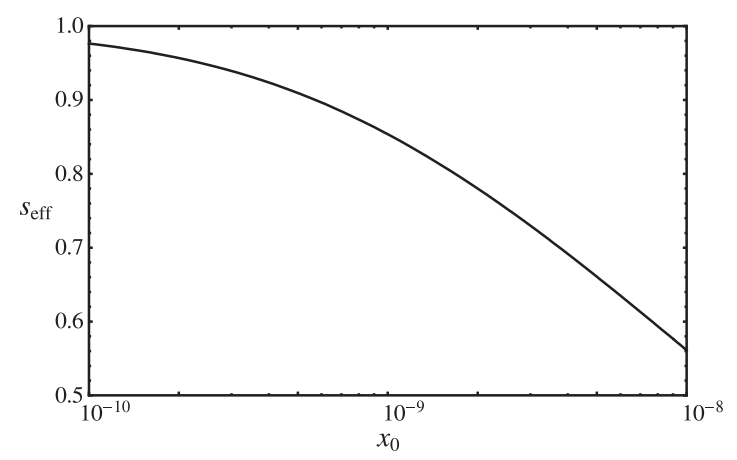

Figure 7. Effective critical exponent of conductivity below $c_{\mathrm{C}}$ as a function of $x_{0}$ for $\delta=0.1\left(c_{\mathrm{C}} \simeq 0.251\right)$ and $x_{2}=5 \cdot 10^{-5}$, calculated with formulas and 3.1 and 3.8 at $c_{1}=0.24$ and $c_{2}=0.25$. 
Our estimates of $s_{\text {eff }}$ with formulas (3.1) and 3.8 are shown in figure 7 They correlate well with typical theoretical [26, 27] and experimental [22] values of 0.75 and $0.7 \div 1.0$, respectively.

\subsection{Behavior of the permittivity}

According to equation (3.2), the effective permittivity is given by

$$
\varepsilon^{\prime}=x \frac{(1-\phi) \varepsilon_{0}^{\prime}+c \frac{\left(2 x+x_{0}\right)^{2}}{(2 x+1)^{2}} \varepsilon_{1}^{\prime}+(\phi-c) \frac{\left(2 x+x_{0}\right)^{2}}{\left(2 x+x_{2}\right)^{2}} \varepsilon_{2}^{\prime}}{(1-\phi) x_{0}+c \frac{\left(2 x+x_{0}\right)^{2}}{(2 x+1)^{2}}+(\phi-c) \frac{\left(2 x+x_{0}\right)^{2}}{\left(2 x+x_{2}\right)^{2}} x_{2}} .
$$

For a badly-conducting matrix $\left(x_{0} \rightarrow 0\right)$ and under the condition $x \ll 1$, three particular situations are of interest to point out to.

1. The system is below the percolation threshold and the conditions $x \ll \sqrt{x_{0}}, x \ll \sqrt{x_{0} x_{2}}, x \ll x_{2}$ (that is, $\sigma \ll \sqrt{\sigma_{0} \sigma_{1}}, \sigma \ll \sqrt{\sigma_{0} \sigma_{2}}$, and $\sigma \ll \sigma_{2}$ ) are met. Then, the dominant contributions to both the numerator and the denominator are made by their first terms, and we expect that $\varepsilon^{\prime} \propto x \propto$ $\left(c_{\mathrm{c}}-c\right)^{-s_{\mathrm{eff}}}$.

2. The system is above the threshold and $x \gg \sqrt{x_{0}}, x \gg \sqrt{x_{2}}, x \gg x_{2}\left(\sigma \gg \sqrt{\sigma_{0} \sigma_{1}}, \sigma \gg \sqrt{\sigma_{1} \sigma_{2}}\right.$, and $\sigma \gg \sigma_{2}$ ). Now, of significance become the first and the third terms in the numerator, the latter being almost independent of $x$, and the second term in the denominator. Correspondingly, the $\varepsilon^{\prime}$ versus $c$ dependence is expected to be close to $\varepsilon^{\prime} \propto x^{-1} \propto\left(c-c_{\mathrm{c}}\right)^{-t_{\text {eff }}}$, with the proportionality constant slightly dependent on $c$.

The exponents $s_{\text {eff }}$ and $t_{\text {eff }}$ in the two preceding scaling-like laws are independent of the components' permittivities $\varepsilon_{i}^{\prime}$.

3. The system is close to the percolation threshold, $x \gg \sqrt{x_{0}}$, and $x \gg x_{2}\left(\sigma \gg \sqrt{\sigma_{0} \sigma_{1}}, \sigma \gg \sigma_{2}\right)$. Then, the numerator is almost $x$-independent, whereas the denominator is mainly contributed to by the second and the third terms. The dependence of $\varepsilon^{\prime}$ on $x$ takes the form $\varepsilon^{\prime} \propto a x /\left(1+b x^{2}\right)$, where the coefficients $a$ and $b$ are easy to recover.

\subsection{Applicability to real systems}

Figure 8 shows the results of processing with formula [3.9] the experimental data [28] for the effective permittivity of the composites prepared by embedding spherical Ag particles (a mean radius $\approx 100 \AA$ ) into a $\mathrm{KCl}$ matrix. The particles were made by evaporating Ag in the presence of argon and oxygen gases so as to form a thin (according to the authors, of approximately $10 \AA, \delta \simeq 0.10$ ) oxide coating on them. This coating prevented the particles from cold-welding together, but was thin enough to allow metal-to-metal contact under high pressure. The composites were prepared by mixing Ag particles and $\mathrm{KCl}$ powder and then compressing the mixture into a solid pellet under high pressure.

It is seen from figure 8 that formula [3.9 not only reproduces data [28] over the entire range of $\mathrm{Ag}$ concentrations investigated, but also gives an estimate of $\delta \simeq 0.14 \div 0.19$, sufficiently close to the expected one.

The conductivity (resistivity $\rho$ ) data for a few $\mathrm{Ag}-\mathrm{KCl}$ composite samples, prepared in the above way, are given in [29]. They pertain only to a very narrow vicinity of the percolation threshold, where $\rho$ drops 7 orders of magnitude with a 1\% Ag volume concentration increment; the parameters of the $\mathrm{KCl}$ matrix are not specified. As figure 9 reveals, formula [3.1] can reproduce data [29] sufficiently well. Better fits can be produced by introducing $c$-dependences for some of the parameters of the model. These facts may indicate that, in addition to experimental errors, various other factors and phenomena (inaccuracy of the function $\phi=\phi(c, \delta)$, particles' size distribution, silver dissolution and local dielectric breakdown in the $\mathrm{KCl}$ matrix, polarization effects, etc.) come into play as $c_{\mathrm{c}}$ is approached. The analysis of them goes far beyond the scope of this paper. 


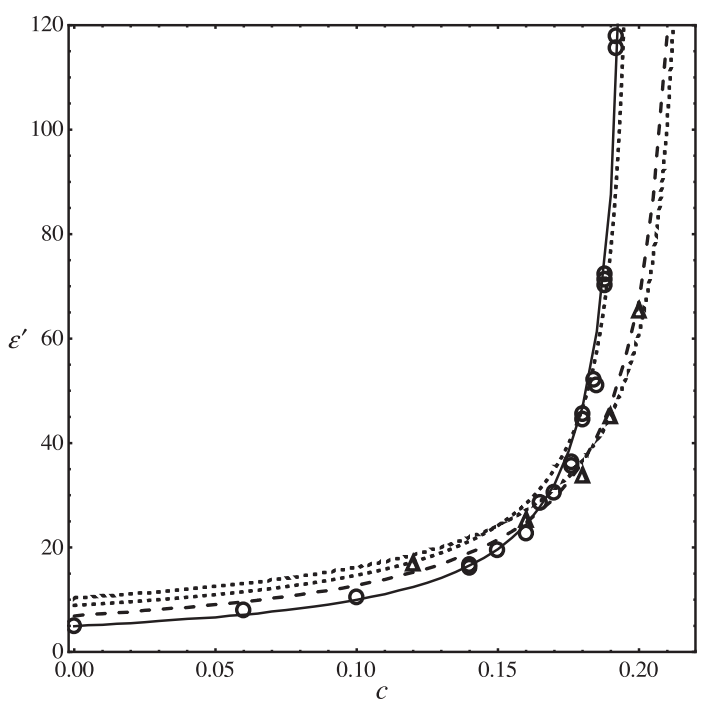

Figure 8. Effective permittivity data [28] for two series of Ag-KCl composite samples (circles and triangles) below the percolation threshold and their fits with formula 3.9 at $\varepsilon_{0}^{\prime}=5.0, \delta=0.186$ (solid line) and $\varepsilon_{0}^{\prime}=7.0, \delta=0.145$ (dashed line). The dotted lines are the scaling-type fits (with $c_{\mathrm{C}}=0.20, s_{\mathrm{eff}}=0.72$ and $c_{\mathrm{c}}=0.22, s_{\mathrm{eff}}=0.74$, respectively) proposed in [28] to the data for $c>0.11$.

\section{6. “Double" percolation}

For intermediate values of $x_{2}\left(x_{0} \ll x_{2} \ll x_{1}\right)$, a "double" percolation can be noticeable, that is, a new increase in $x$ after some levelling off (figure 2); it is accompanied by the appearance of a new peak in the concentration dependence of the permittivity (figure 10. The physical cause of this phenomenon is clear - in a concentrated system, the hard cores of particles with penetrable shells begin to contact intensively to form percolation cluster and add to the effective conductivity.

Evidently, the threshold concentration $c_{c}^{\prime}$ for "double" percolation is close to a value of $1 / 3$. In the region $\left|c-\frac{1}{3}\right| \ll x_{2} \ll 1$, the $c$-dependence of the effective conductivity (3.3) is represented by the squareroot law

$$
x=\frac{1}{2}\left(3 x_{2}\right)^{1 / 2}\left[\phi(c, \delta)-\frac{1}{3}\right]^{1 / 2}+O\left(x_{2}\right)=\frac{1}{2}\left[3 x_{2} \phi^{\prime}\left(c_{\mathrm{c}}, \delta\right)\right]^{1 / 2}\left(c-c_{\mathrm{c}}\right)^{1 / 2}+O\left(x_{2}\right),
$$

$\phi^{\prime}$ being the derivative of $\phi$ with respect to $c$. For concentrations satisfying the condition $c-\frac{1}{3} \gg x_{2}$, it

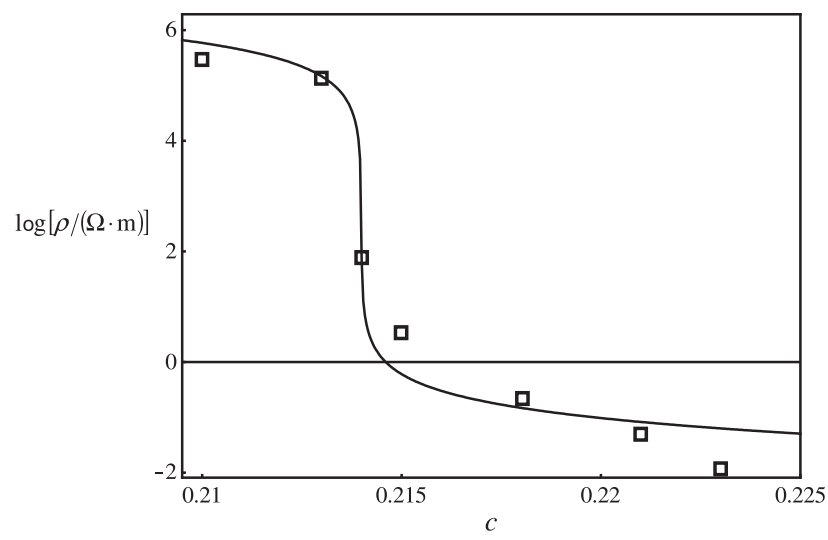

Figure 9. Effective resistivity data [29] for $\mathrm{Ag}-\mathrm{KCl}$ composite samples (squares) and the fit to them with formula 3.1] at $\sigma_{1}=6.3 \cdot 10^{7} \mathrm{~S} / \mathrm{m}, x_{0}=5 \cdot 10^{-16}, \delta=0.162\left(c_{\mathrm{C}}=0.214\right)$, and $x_{2}=4 \cdot 10^{-6}$. 


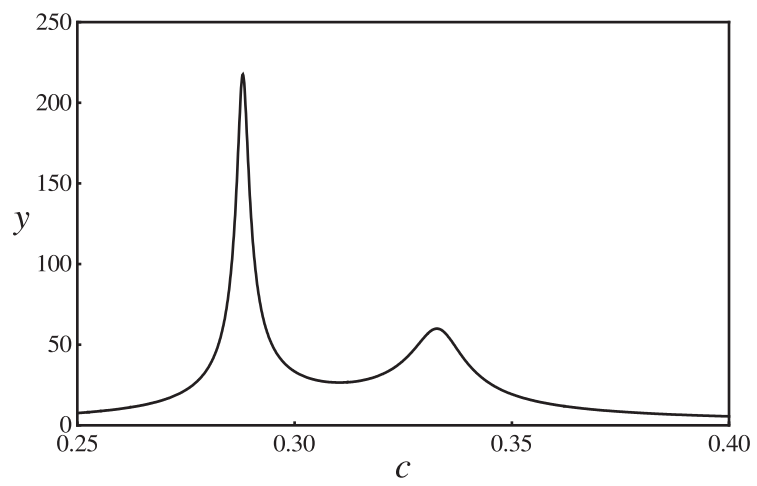

Figure 10. Effective permittivity as "double" percolation occurs; $x_{0}=1 \cdot 10^{-8}, x_{2}=5 \cdot 10^{-4}, y_{1}=1.5, y_{2}=1$, $\delta=0.05$.

becomes linear,

$$
x=\frac{3}{2}\left(c-\frac{1}{3}\right)+O\left(x_{2}\right),
$$

with a considerably greater amplitude as compared to those in formulas (3.6) and (3.10).

As for now, we are unaware of experimental observations of the effect described. Usually, "double" percolation is associated with a non-monotonous behavior of the conductivity in composites made by embedding the conducting particles into a two-component matrix (see, for example, [30, 31]).

It can also be shown that a decrease in $x$ can occur at $c \sim c_{\mathrm{c}}^{\prime}$ in systems with $x_{2} \gg 1$. Similar effects were observed in two-phase composite solid electrolytes with a highly conducting interphase layer [22].

\section{Concluding remark}

The model proposed is interesting in the sense that it is based on rather clear assumptions, incorporates many-particle effects in a consistent way, and can be further refined so as to apply to a more complicated systems, including fluctuation phenomena, etc. For practice, it can serve as a rather flexible theoretical basis for analysis of dielectric and conductive properties of such complex systems as various dispersions, colloids, and so-called nanofluids, or for the development of new composite materials.

\section{Acknowledgement}

We are grateful to an anonymous Referee for stimulating remarks.

\section{References}

1. Cairns D., Armes S., Bremer L., Langmuir, 1999, 15(23), 8052; doi 10.1021/la990442s

2. Ranjbar Z., Rastegar S., Colloids Surf., A, 2006, 290, 186; doi 10.1016/j.colsurfa.2006.05.025

3. Yan S., Zhen L., Xu C.Y., Jiang J.T., Shao W.Z., J. Phys. D: Appl. Phys., 2010, 43, 245003; doi $10.1088 / 0022-3727 / 43 / 24 / 245003$

4. Liu X., Wu Y., Wang X., Li R., Zhang Z., J. Phys D: Appl. Phys., 2011, 44, 115402; doi $10.1088 / 0022-3727 / 44 / 11 / 115402$

5. Kirkpatrick S., Rev. Mod. Phys., 1973, 45, 574; doi 10.1103/RevModPhys.45.574.

6. Clerk J., Giraud G., Laugier J., Luck J., Adv. Phys., 1990, 39, 191; doi10.1080/00018739000101501

7. Stauffer D., Aharony A., Introduction to Percolation Theory (2nd revised ed.), Taylor \& Francis, 2003.

8. Pike G., Seager C., Phys. Rev. B, 1974, 10, 1421; doi 10.1103/PhysRevB.10.1421

9. Seager C., Pike G., Phys. Rev. B, 1974, 10, 1435; doi 10.1103/PhysRevB.10.1435

10. Callaway D., Newman M., Strogatz S., Watts D., Phys. Rev. Lett., 2000, 85, 5468; doi 10.1103/PhysRevLett.85.5468

11. Maxwell-Garnett J., Philos. Trans. R. Soc. London, Ser. A, 1904, 203, 359; doi 10.1098/rsta.1904.0024 
12. Bruggeman D., Ann. Phys., 1935, 24, 636; doi 10.1002/andp.19354160705

13. Snarskii A., Usp. Fiz. Nauk, 2007, 177, 1341 (in Russian); doi 10.3367/UFNr.0177.200712g.1341 [Physics-Uspekhi, 2007, 50, 1239; doi 10.1070/PU2007v050n12ABEH006348].

14. Kigami J., Analysis on Fractals, Cambridge University Press, 2001.

15. McLachlan D., J. Phys. C: Solid State Phys., 1986, 19, 1339; doi 10.1088/0022-3719/19/9/007

16. Sushko M.Ya., Zh. Eksp. Teor. Fiz., 2007, 132, 478 (in Russian) [JETP, 2007, 105, 426; doi 10.1134/S1063776107080146].

17. Sushko M.Ya., Kriskiv S., Zh. Tech. Fiz., 2009, 79, 97 (in Russian) [Tech. Phys., 2009, 54, 423; doi 10.1134/S1063784209030165].

18. Sushko M.Ya., J. Phys. D: Appl. Phys., 2009, 42, 155410; doi 10.1088/0022-3727/42/15/155410.

19. Rikvold P., Stell G., J. Colloid Interface Sci., 1985, 108, 158; doi 10.1016/0021-9797(85)90246-2

20. Rottereau M., Gimel J., Nicolai T., Duran. D., Eur. Phys. J. E, 2003, 11, 61; doi 10.1140/epje/i2003-10006-x

21. Torquato S., J. Chem. Phys., 1984, 81, 5079; doi 10.1063/1.447497

22. Nan C.-W., Prog. Mater Sci., 1993, 37, 1; doi 10.1016/0079-6425(93)90004-5

23. Landau L.D., Lifshitz E.M., Pitaevskii L.P., Electrodynamics of Continuous Media. Vol. 8, Nauka, 1984.

24. Korn G., Korn T., Mathematical Handbook, McGrew-Hill Book Company, New York, 1968.

25. Efros A., Shklovskii B., Phys. Status Solidi B, 1976, 76, 475; doi 10.1002/pssb.2220760205

26. Bernasconi J., Phys. Rev. B, 1978, 18, 2185; doi 10.1103/PhysRevB.18.2185.

27. Luck J.M., J. Phys. A: Math. Gen., 1985, 18, 2061; doi 10.1088/0305-4470/18/11/027

28. Grannan D., Garland J., Tanner D., Phys. Rev. Lett., 1981, 46, 375; doi 10.1103/PhysRevLett.46.375

29. Chen I.-G., Johnson W., J. Mater. Sci., 1986, 21, 3162; doi 10.1007/BF00553352.

30. Al-Saleh M., Sundararaj U., Eur. Polym. J., 2008, 44, 1931; doi 10.1016/j.eurpolymj.2008.04.013

31. Konishi Y., Cakmak M., Polymer, 2006, 47, 5371; doi 10.1016/j.polymer.2006.05.015

\title{
Провідність та діелектрична проникність дисперсних систем із вільно-проникним міжфазним шаром між частинками й середовищем
}

\author{
М.Я. Сушко, А.К. Семенов \\ Одеський національний університет імені I. І. Мечникова, вул. Дворянська, 2, 65026 Одеса, Україна \\ Запропоновано модель для вивчення ефективних квазістатичних провідності та діелектричної проник- \\ ності дисперсних систем із вільно-проникним міжфазним шаром між частинками та середовищем, яка \\ ефективно враховує багаточастинкові поляризаційні та кореляційні ефекти. Структура компонентів сис- \\ теми, включаючи міжфазний шар, враховується шляхом моделювання профілів їх низькочастотної комп- \\ лексної діелектричної проникності. Модель, зокрема, описує перколяційно-подібну поведінку ефективної \\ провідності, що супроводжується суттєвим зростанням дійсної частини ефективної комплексної проник- \\ ності системи. Положення порогу перколяції визначається головним чином товщиною міжфазного шару. \\ Передбачено ефект “подвійної” перколяції. Результати порівняно з експериментом.
}

Ключові слова: структурована частинка, дисперсна система, діелектрична проникність, провідність, перколяція 